\title{
ORIGINAL ARTICLE \\ Central motor and sensory conduction in patients with hepatic myelopathy
}

\author{
R Nardone ${ }^{1,2,3}$, A Orioli ${ }^{2}$, Y Höller ${ }^{1,3}$, P Lochner $^{2}$, A Kunz ${ }^{1}$, S Golaszewski ${ }^{1}$, F Brigo ${ }^{2,4}$ and E Trinka ${ }^{1,3}$
}

Study design: Experimental neurophysiological study.

Objectives: The hepatic myelopathy (HM) is characterized by progressive weakness and spasticity of the lower extremities, while there are only a few reports of sensory impairment. However, sensory function has been poorly explored in HM. We believe that an electrophysiological assessment of dorsal columns by somatosensory evoked potentials (SEPs) and of cortico-spinal lateral tracts by motor evoked potentials (MEPs) should be of considerable value in the functional evaluation of the spinal cord involvement in patients with HM.

Setting: Salzburg (Austria) and Merano (Italy).

Methods: Eight patients diagnosed with HM were studied with MEPs and SEPs. Neurological examination revealed different degrees of cortico-spinal tract involvement in all patients and sensory abnormalities in three patients.

Results: Central motor conduction to lower limb muscles was abnormal in all patients, while central sensory conduction was abnormal in seven out of the eight patients. Both central motor and sensory conduction to upper limbs are normal in all patients.

Conclusion: The main finding is that electrophysiological evidence of central sensory involvement is present in a very high percentage of patients with $\mathrm{HM}$, and that the threshold for electrophysiological abnormalities is below that for clinical manifestations. Therefore, central sensory and motor conduction studies are sensitive methods for detecting, localizing and monitoring spinal cord damage in HM.

Spinal Cord (2014) 52, 439-443; doi:10.1038/sc.2014.61; published online 29 April 2014

\section{INTRODUCTION}

A severe spinal cord involvement may rarely occur in patients with cirrhosis and other chronic liver diseases; this complication is usually associated with overt liver failure and surgical or spontaneous portosystemic shunt. The hepatic myelopathy (HM) is characterized by progressive weakness and spasticity of the lower extremities, while there are only a few reports of sensory or sphincter impairment. ${ }^{1-4}$ Motor evoked potentials (MEPs) studies disclosed MEP abnormalities even in patients with normal clinical examination. ${ }^{5}$ However, sensory function has poorly been explored in HM patients.

Somatosensory evoked potentials (SEPs) are a widely used and high sensitive method of detecting disturbances of central sensory conduction. To the best of our knowledge, SEP studies have never been performed in patients with HM.

The aim of this study was to explore central motor and sensory function in patients with HM objectively using MEPs as well as upper and lower limb SEPs.

\section{MATERIALS AND METHODS}

\section{Patients}

We studied eight patients with HM (three women and five men, mean age 60.8 years, range $44-75$ years). The clinical and demographic data of the patients are shown in Table 1.
All the patients exhibited clear-cut signs of spinal cord involvement and three of them exhibited varying degrees of disability. Two patients had mild neurological abnormalities (hyperreflexia, extensor plantar responses) without disability, three patients experienced minimal disability (stiffness, nocturnal spasms and leg cramps, and three patients mild to severe paraparesis.

Neurological examination revealed sensory abnormalities in three patients. They showed loss of proprioception and vibratory sensation. Another patient complained of intermittent paraesthesias in the feet without sensory deficits.

Brain and spinal magnetic resonance imaging was unremarkable in all patients.

Nerve conduction studies provided no evidence of polyneuropathy in all patients.

The diagnosis of HM was established on clinical grounds after exclusion of the other possible causes. Peripheral blood cell count, serum values for renal function tests, calcium and phosphate were normal in all patients; creatine kinase was slightly increased in two patients. Specific laboratory tests have been performed to exclude a metabolic/nutritional disorders (vitamin B12, vitamin E, folate or copper deficiency, latyrism), viral infections (varicella zoster virus, Epstein Barr virus, herpes simplex virus, cytomegalovirus, myelopathy associated with acquired immunodeficiency syndrome and human T-cell lymphotropic virus type I-associated myelopathy/tropical spastic paraparesis), fungal infection (cryptococcus, apergillus), neuroborreliosis, neurosyphilis and, in four patients also rare metabolic diseases, such as adrenoleucodystrophy, heroxaminidase deficiency or cerebrotendinous xanthomatosis.

Liver function was reduced in all patients; plasma ammonia level was elevated in six patients and normal in two patients.

${ }^{1}$ Department of Neurology, Center for Cognitive Neuroscience, Christian Doppler Klinik, Paracelsus Medical University, Salzburg, Austria; ${ }^{2}$ Department of Neurology, Franz Tappeiner Hospital, Merano, Italy; ${ }^{3}$ Spinal Cord Injury and Tissue Regeneration Center, Paracelsus Medical University, Salzburg, Austria and ${ }^{4}$ Section of Clinical Neurology, Department of Neurological, Neuropsychological, Morphological and Movement Sciences, University of Verona, Verona, Italy Correspondence: Dr R Nardone, Department of Neurology, Franz Tappeiner Hospital, Via Rossini, 5, Merano (BZ) 39012, Italy. E-mail: raffaele.nardone@asbmeran-o.it

Received 7 February 2014; revised 17 March 2014; accepted 28 March 2014; published online 29 April 2014 
Table 1 Demographic and clinical characteristics of the patients with hepatic myelopathy

\begin{tabular}{|c|c|c|c|c|c|c|}
\hline Patients & Age & Gender & Liver disease & Ammonia ${ }^{a}$ & Motor signs/symptoms & Sensory signs/symptoms \\
\hline 1 & 55 & M & Posthepatitic cirrhosis & 75 & Hyperreflexia, Babinski sign & None \\
\hline 2 & 44 & M & Postalcoholic cirrhosis & 53 & Hyperreflexia, Babinski sign & None \\
\hline 3 & 69 & $\mathrm{~F}$ & Postalcoholic cirrhosis & 88 & $\begin{array}{l}\text { Hyperreflexia, Babinski sign, } \\
\text { stiffness, legs cramps }\end{array}$ & None \\
\hline 4 & 67 & $\mathrm{~F}$ & Postalcoholic cirrhosis & 84 & $\begin{array}{l}\text { Hyperreflexia, Babinski sign, } \\
\text { stiffness, legs cramps }\end{array}$ & None \\
\hline 5 & 75 & M & Posthepatitic Cirrhosis & 45 & $\begin{array}{l}\text { Hyperreflexia, Babinski sign, } \\
\text { stiffness, nocturnal spasms }\end{array}$ & Subjective paraesthesias in the feet \\
\hline 6 & 49 & $\mathrm{~F}$ & Posthepatitic cirrhosis & 65 & Mild paraparesis & $\begin{array}{l}\text { Subjective distal paraesthesias Proprio- } \\
\text { ception and vibratory sensory loss }\end{array}$ \\
\hline 7 & 60 & M & Postalcoholic cirrhosis & 102 & Moderate paraparesis & Proprioception and vibratory sensory loss \\
\hline 8 & 68 & M & Posthepatitic cirrhosis & 82 & Severe paraparsis & $\begin{array}{l}\text { Subjective distal paraesthesias proprio- } \\
\text { ception and vibratory sensory loss }\end{array}$ \\
\hline
\end{tabular}

*Plasma ammonia level $\left(\mu \mathrm{mol} \mathrm{I}^{-1}\right)$, bold type indicates abnormal values.

In three patients, the cerebrospinal fluid had also been examined; the analysis revealed normal findings with the exception of a slightly increased protein concentration in one patient.

Thirteen age-matched healthy volunteers (five women and eight men; mean age 58.8 years, range $40-76$ years) represented the control population.

Patients and healthy volunteers provided informed consent before participation in this study, which was performed according to the Declaration of Helsinki.

\section{Motor evoked potentials}

Central motor conduction was evaluated using magnetic stimulation of the motor cortex and spine through a $120-\mathrm{mm}$ coil powered by a High-power Magstim 200 Stimulator (Magstim Co, Dyfed, UK). Responses were recorded from the contralateral abductor digiti minimi and tibialis anterior muscles by 9-mm diameter $\mathrm{Ag}-\mathrm{AgCl}$ surface electrodes and amplified with filter settings of 2 and $5 \mathrm{~Hz}$. The maximum magnetic field generated was $\sim 2 \mathrm{~T}$ at the center of the coil. The stimulus intensity was $100 \%$ of the maximum output for cortical stimulation. The coil was centered over the vertex or $2-3 \mathrm{~cm}$ anterior to the vertex to achieve maximal responses for the upper and the lower limbs, respectively. To obtain preferential activation of each hemisphere, a clockwise inducing current flow, as viewed from above, was used for the right motor cortex and an anticlockwise flow for the left motor cortex. Cortical stimulation was performed during voluntary contraction of the tested muscle of about $20 \%$ of maximum voluntary contraction to facilitate the responses.

The central motor conduction time (CMCT) was calculated by subtracting the peripheral conduction time from spinal cord to muscles from the latency of responses evoked by cortical stimulation. ${ }^{6}$ To evaluate peripheral motor conduction from the spinal cord to the muscles, magnetic stimulation on the cervical and lumbar spine was performed. For the radicular stimulation, the lower edge of the coil was placed just laterally to C7-D1 and L4-L5 spinous processes for abductor digiti minimi and tibialis anterior muscles, respectively. A clockwise inducing current, as viewed from behind, was used for the right muscles and vice versa from the left muscles. The stimulus intensity was $60 \%$ of maximum output. Peripheral conduction time was also calculated from $\mathrm{F}$ wave recordings, as $(F+M-1) / 2$, where $F$ is the shortest F-latency and $M$ is the M-wave latency. ${ }^{7}$

Normal limits for CMCT were defined by mean \pm 3 s.d.s of the control values.

\section{Somatosensory evoked potentials}

For SEP recording (Sensor, Medelec, Surrey, UK) the patient lay on a couch in a warm and semi-darkened room. Stimuli $(0.3 \mathrm{~ms}$ square pulses) were delivered at a rate of $5 \mathrm{~Hz}$ at motor threshold intensity. Stimulations were delivered at the wrist for median nerve SEPs and at the ankle for tibial nerve SEPs. The filter bandpass was $10-3000 \mathrm{~Hz}$; the analysis time was $50 \mathrm{~ms}$ for
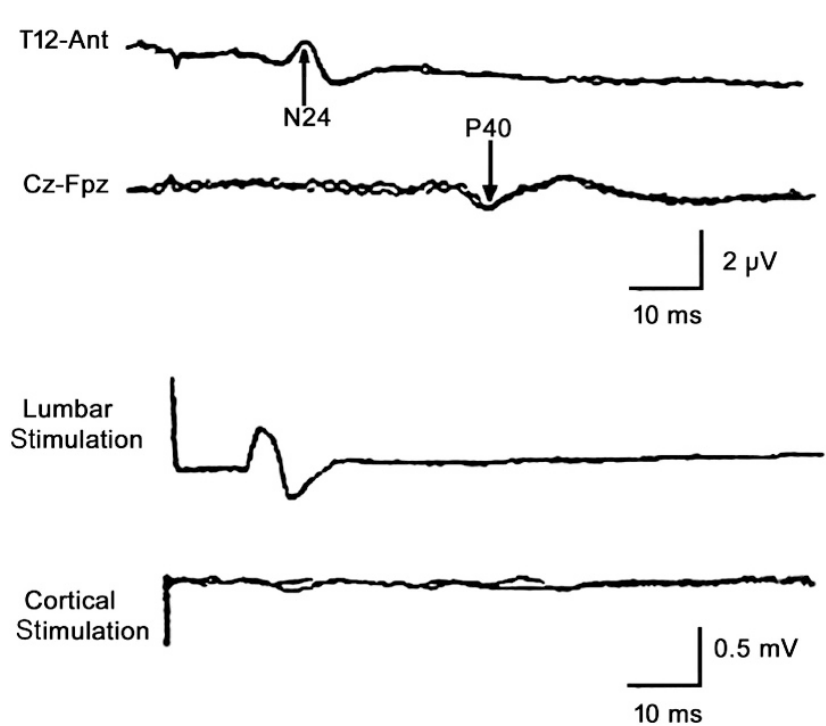

Figure 1 Patient 8. SEPs evoked by right tibial nerve stimulation (upper traces) and MEPs from tibialis anterior muscle evoked by lumbar and cortical stimulation (lower traces). SEPs: the latency of N24 response is within normal limits, while the latency of the P40 response and the N24P40 interpeak interval are prolonged. MEPs: the latency of the response obtained after radicular stimulation is normal, while no response is obtained after cortical stimulation.

median nerve SEPs and $100 \mathrm{~ms}$ for tibial nerve SEPs. Two averages of 2048 trials each were obtained and drawn out by computer on an $\mathrm{x}-\mathrm{Y}$ plotter.

For median nerve SEP recording, the electrodes (impedence below $5 \mathrm{k} \Omega$ ) were placed in the supraclavicular fussa (Erb's point), over the spinous process of the sixth cervical vertebra (Cv6) and in the contralateral and ipsilateral parietal scalp regions. The Erb's point electrode was referred to $\mathrm{Fz}$ and the parietal scalp electrodes to the shoulder contralateral to the stimulated side. To assess conduction in the dorsal column system and in the intracranial segments the P9-P14 and P14-N20 interpeak latencies were measured. The P14 and N20 potentials were measured on the contralateral parietal traces recorded with a shoulder reference montage. ${ }^{8}$

For tibial nerve SEP recording, the electrodes were placed over the spinous process of the 12th thoracic vertebra (Th12) ad on the vertex (Cz). The $\mathrm{Cz}$ electrode was referred to the forehead (Fpz) to record the P40 cortical response. ${ }^{9,10}$ To calculate the central somatosensory conduction, the N24-P40 interpeak interval was measured. 
For recording of the spinal N24 potential, we connected grid 1 of the amplifier to the Th12 electrode and grid 2 to an electrode located in the anterior region of the abdomen immediately above the umbilicus (Ant).

\section{RESULTS}

\section{MEP findings}

MEP findings were abnormal in $8 / 8$ patients (100\%). The abnormalities were always bilateral and involved the lower limbs. CMCT was prolonged in six patients and cortical responses were absent in two patients (Figure 1). Peripheral motor conduction was always normal.
MEP data of patients and control subjects are summarized in Table 2.

\section{SEP findings}

SEPs were abnormal in $7 / 8$ patients $(87 \%)$. The abnormalities were always bilateral and represented by a prolonged N24-P40 interval (Figure 1). Median SEPs were always normal. Peripheral components of SEPs were of normal latency in all patients.

SEP data of patients and control subjects are summarized in Table 3 .

\section{Table 2 Motor evoked potentials findings}

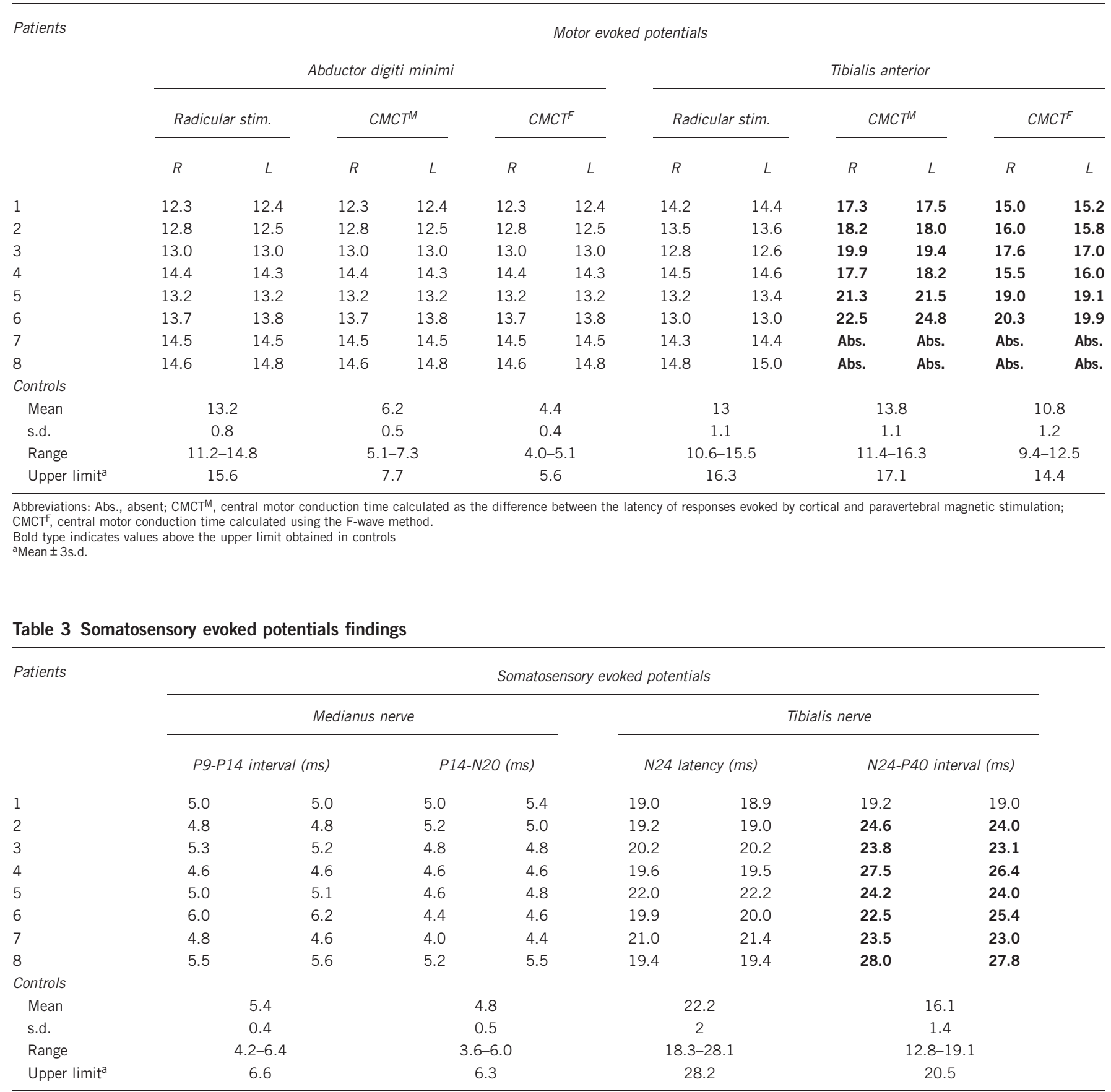

Bold type indicates values above the upper limit obtained in controls. 


\section{DISCUSSION AND CONCLUSIONS}

The present study is the first that electrophysiologically characterizes the functional involvement of both, motor and sensory central pathways, in HM using SEPs and MEPs. Sensory disturbances have rarely been described in $\mathrm{HM}$ and are thought to usually less important.

However, the main finding of our study was that we documented, beside the expected central motor conduction abnormalities, a significant involvement of the central sensory pathways in patients with HM.

Moreover, electrophysiological evaluation of central sensory conduction may disclose an impairment of the central sensory pathways even before the sensory disturbances are clinically manifest.

Our results are consistent with previous studies demonstrating pathological findings in the posterior columns of HM patients. ${ }^{4,11}$ The histology of HM consists of symmetrical loss of myelin in the lateral pyramidal tracts, that begins with demyelination beginning in the cervical spine, becoming more intense at lower levels, and occasionally being associated with axonal damage. ${ }^{12,13}$ In the early stages, demyelination seems to predominate, but as the disease progresses axonal loss occurs, and this is likely to be irreversible. ${ }^{2,14}$ Occasionally, demyelination has also been found in the ventral pyramidal tracts, in the posterior columns and spinocerebellar tracts. A recent study also documented in an HM patient a delayed onset posterior column dysfunction (proprioception and vibratory sensory loss) and a small fiber length-dependent axonal polyneuropathy, ${ }^{15}$ both progressing concomitantly with the motor deficits.

The pathogenesis of HM is not yet understood, but there is a close relationship between an extensive portosystemic shunt and the occurrence of $\mathrm{HM}$, even in the absence of liver dysfunction. ${ }^{16}$ It can thus be hypothesized that the shunting of blood may allow nitrogenous breakdown products or a neurotoxin to bypass the liver and damage the spinal cord. In particular, nitrogenous products such as ammonia have been identified as a major contributor to the development of HM. ${ }^{17}$

Interestingly, Nardone et al. ${ }^{5}$ found that clinical and MEP results of patients with a more advanced stage of disease (with markedly prolonged CMCT) were not substantially altered by liver transplantation, whereas patients with strong evidence of early-stage disease (minor CMCT abnormalities) exhibited clear neurophysiological and clinical improvement after liver transplantation. These MEP findings suggest that HM may be reversible if treated in the early stages of the disease, ${ }^{18,19}$ whereas spinal cord dysfunction remains unchanged or is less likely to be reversed by liver transplantation in the later stages. ${ }^{20}$ Therefore, early diagnosis of $\mathrm{HM}$ and subsequent liver transplantation have to be recommended.

Obviously, demyelinated axons within the spinal cord can be well visualized by modern neuroimaging techniques, in particular the diffusion tensor tractography in MRI. However, diffusion tensor tractography is an expensive technique that is still not commonly and routinely used for clinical applications, while SEP and MEP studies can be easily performed in the most laboratories of neurophysiology. Moreover, the neurophysiological evaluation is essential to provide functional correlate to radiological abnormalities.

The results of the present study provide electrophysiological evidence that posterior column spinal cord pathology is more common in HM than previously recognized.

Utku et al. performed an MEP study in two patients and found an absence of cortical MEPs in both the lower and upper extremities, and postulated that the lesion was localized at the cervical level of the spinal cord. ${ }^{21}$ In contrast, Nardone et al. ${ }^{5}$ found an abnormal CMCT to the lower lumbar spinal segments and a normal CMCT to the upper cervical spinal segments, thus supporting localization of main dysfunction to the thoracic spinal cord. Moreover, another MEP study of HM patients indicated that the sites of higher vulnerability are located between the upper thoracic and the lumbar spinal cord. ${ }^{22}$

In a recently reported patient the abnormal MRI finding of increased fluid-attenuated inversion recovery signal in the subcortical white matter and subcortical spinal tracts suggests the possible occurrence of HM-related pathology above the level of the foramen magnum. ${ }^{15}$ We cannot find any MRI abnormalities in our patients. Moreover, the normality of the N24-P40 interval also excludes a subclinical involvement of the intracranial segment of the central sensory pathways in our patients.

The pronounced slowing of central conduction is similar to that described in multiple sclerosis patients, ${ }^{23,24}$ and is thus compatible with demyelination in central sensory and motor pathways.

Our SEP findings suggest that in HM the thoracic cord is usually affected first and confirm that central sensory and motor conduction studies are sensitive methods for detecting such damage.

In conclusion, an electrophysiological assessment of dorsal columns by SEPs and of cortico-spinal lateral tracts by MEPs should be of considerable value in the functional evaluation of the spinal cord involvement in the patients affected by HM.

\section{DATA ARCHIVING}

There were no data to deposit.

\section{CONFLICT OF INTEREST}

The authors declare no conflict of interest.

1 Zieve L, Mendelson DF, Goepfert M. Shunt encephalomyelopathy. II. Occurrence of permanent myelopathy. Ann Intern Med 1960; 53: 53-63.

2 Liversedge LA, Rawson MD. Myelopathy in hepatic disease and portosystemic venous anastamosis. Lancet 1966; 1: 277-279.

3 Bain VG, Bailey RJ, Jhamandas JH. Postshunt myelopathy. J Clin Gastroenterol 1991; 13: 562-564.

4 Leigh AD, Card WI. Hepato-lenticular degeneration. A case associated with posteriorlateral column degeneration. J Neuropathol Exp Neurol 1949; 8: 338.

5 Nardone R, Buratti T, Oliviero A, Lochmann A, Tezzon F. Corticospinal involvement in patients with a portosystemic shunt due to liver cirrhosis: a MEP study. J Neurol 2006 253: 81-85.

6 Rossini PM, Barker AT, Berardelli A, Caramia MD, Caruso G, Cracco RQ et al. Non invasive electrical and magnetic stimulation of the brain, spinal cord and roots: basic principles and procedures for routine clinical application: Report of IFCN committee. Electroencephalogr Clin Neurophysiol 1994; 91: 79-92.

7 Mills KR. Magnetic Stimulation of the Human Nervous System. Oxford University Press: Oxford, 1999.

8 Tsuji S, Shibasaki H, Kato M, Kuroiwa Y, Shima F. Subcortical thalamic and cortical somatosensory evoked potentials to median nerve stimulation. Electroencephalogr Clin Neurophysiol 1984; 59: 465-476.

9 Jones SJ, Small DG. Spinal and sub-cortical potentials following stimulation of the posterior tibial nerve in man. Electroencephalogr Clin Neurophysiol 1978; 44 299-306.

10 Kakigi R, Shibasaki H, Hashizume A, Kuroiwa Y. Short latency somatosensory evoked spinal and scalp-recorded potentials following posterior tibila nerve stimulation in man. Electroencephalogr Clin Neurophysiol 1982; 53: 602-611.

11 Mendoza G, Marti-Fabregas J, Kulisevsky J, Escartin A. Hepatic myelopathy: a rare complication of portacaval shunt. Eur Neurol 1994; 34: 209-212.

12 Pant SS, Rebeiz JJ, Richardson EP Jr.. Spastic paraparesis following portocaval shunts. Neurology 1968; 18: 135-141.

13 Campellone JV, Lacomis D, Giuliani MJ, Kroboth FJ. Hepatic myelopathy. Case report with review of the literature. Clin Neurol Neurosurg 1996; 98: 242-246.

14 Lewis M, Howdle PD. The neurology of liver failure. Q J Med 2003; 96 623-633. 
15 Caldwell C, Werdiger N, Jakab S, Schilsky M, Arvelakis A, Kulkarni S et al. Use of model for end-stage liver disease exception points for early liver transplantation and successful reversal of hepatic myelopathy with a review of the literature. Liver Transpl 2010; 16: 818-826.

16 Troisi R, Debruyne J, de Hemptinne B. Improvement of hepatic myelopathy after liver transplantation. N Engl J Med 1999; 340: 151.

17 Weissenborn K, Tietge UJ, Bokemeyer M, Mohammadi B, Bode U, Manns MP et al. Liver transplantation improves hepatic myelopathy: evidence by three cases. Gastroenterology 2003; 124: 346-351.

18 Counsell C, Warlow C. Failure of presumed hepatic myelopathy to improve after liver transplantation. J Neurol Neurosurg Psychiatry 1996; 60: 590.

19 Demirci M, Tan E, Elibol B, Gedikoglu G, Saribas O. Spastic paraparesis associated with portal-systemic venous shunting due to congenital hepatic fibrosis. Neurology 1992; 42: 983-985.
20 Yengue P, Adler M, Bouhdid H, Mavroudakis N, Gelin M, Bourgeois N. Hepatic myelopathy after splenorenal shunting: report of one case and review of the literature. Acta Gastroenterol Belg 2001; 64: 231-233.

21 Utku U, Asil T, Balci K, Uzunca I, Celik Y. Hepatic myelopathy with spastic paraparesis. Clin Neurol Neurosurg 2005; 107: 514-516.

22 Koo JE, Lim YS, Myung SJ, Suh KS, Kim KM, Lee HC et al. Hepatic myelopathy as a presenting neurological complication in patients with cirrhosis and spontaneous splenorenal shunt. Korean J Hepatol 2008; 14: 89-96.

23 Comi G, Leocani L, Medaglini S, Locatelli T, Martinelli V, Santuccio G et al. Evoked potentials in diagnosis and monitoring of multiple sclerosis. Electroencephalogr Clin Neurophysiol Suppl 1999; 49: 13-18.

24 Hess CW, Mills KR, Murray NM, Schriefer TN. Magnetic brain stimulation: central motor conduction studies in multiple sclerosis. Ann Neurol 1987; 22 : 744-745. 